\title{
Reviews on Machine Learning based Adaptive Mobile Learning System
}

\author{
Adeboje Olawale Timothy \\ Computer Science Department, \\ Federal University of Technology, \\ Akure, Nigeria
}

\author{
Jimoh Ibraheem Temitope \\ Computer Science Department, \\ Federal University of Technology, \\ Akure, Nigeria
}

\author{
Isiaka Abdulwab \\ Computer Science Department, \\ Federal College of Agriculture, \\ Akure, Nigeria \\ Joda Shade \\ Computer Science Department, \\ Federal College of Agriculture, \\ Akure, Nigeria
}

\begin{abstract}
It is obvious that the increasing number of new mobile devices has encouraged learners to use these devices to access content without sacrificing usability and accessibility. However, most current learning contents to be accessed are typical designed for desktop computers, hence may not be suitable for presentation on other devices like mobile devices as they are mostly affected with limited bandwidth and limited device capabilities. Notwithstanding, mobile learning researchers are devoted to finding ways of harmonizing device adaptation (device type and capabilities) with content adaptation (that is learner learning style, preference, strategies, and so on) to satisfy individual demands. However, some of the researches attempting to achieve these features are either faced with one challenges or the other. Therefore, this paper is set to provide a proper background on Mobile learning and set to resolve the shortcomings in the reviewed literatures by developing ANFIS based mobile learning system that incorporates an automatic learning style identification module.
\end{abstract}

\section{Keywords}

Machine Learning, Mobile Learning, Adaptive Neuro-Fuzzy Inference System (ANFIS), Felder-Silverman Learning Style Model

\section{INTRODUCTION}

The fast growth of Information and Communication Technologies (ICT) has become one of the essential technological transformations of this modern era as it has contributed greatly to societal economic and social advancement. This changes in society brought about by ICT advances, has equally led to a new type of learning model (mobile-learning) that has no restriction to time and place as compared to electronic-learning that comes before it.

Mobile learning (or m-learning) is one of the types of online learning that cuts across multiple learner contexts delivering content through mobile technologies and the Internet. This type of learning is dynamic because it eases learning capabilities with the opportunity to learn independent of time and location. Mobile learning helps users to learn a course at anytime, anywhere and also provide the unique experience to the learners in terms of its flexibility. Moreover, the increasing ownership of mobile devices and the growth of mobile communications industry have provided a number of services such as instant interpersonal and group communication, location-aware information delivery and personalized assistance based on users' preferences and needs, as well as ubiquitous communication and interaction with smart devices and object [1].

However, new mobile technologies alone cannot improve the experience of mobile learners as there are a number of factors that impact on a typical learning experience such as learning styles, learning model, and strategies [2]. Over the last decades, there has been a growing interest in adaptive and personalized learning by researchers in Technology-enhanced Learning (TeL), which has led to the initiative of incorporating the functionalities of mobile technologies with personalized and adaptive learning paradigm in order to maximizing learner's satisfaction, learning speed and learning effectiveness [1].

Adaptivity and personalization in mobile learning systems refer to the process of enabling the system to fit its behaviour and functionalities to the educational needs (such as learning goals and interests), the personal characteristics (such as learning styles and different prior knowledge) and the particular circumstances (such as the current location and movements in the environment) of the individual learner or the group of interconnected learners [3]. Adaptation deals with taking learners' situation, educational needs and personal characteristics into consideration in generating appropriately designed learning experiences, whereas personalization is a more general term and deals with the customization of the system features, it also include issues which can be adapted and specified by learners themselves, such as the system interface, the preferred language, or other issues which make the system more personal [4].

Two approaches in the field of context-aware adaptive and personalized learning for implementing adaptation engines include: Rule based adaptation and Algorithm based adaptation approaches. The formal involves the use of conditional structures of IF/THEN/ELSE statements for mobile content adaption, while the latter involve the use of algorithms such as heuristic algorithms, similarity algorithms, decision based algorithms, and more for mobile content adaption. Rule based system are content oriented and easier to understand in terms of functionalities, but are limited in their ability to adapt individual learner's abilities or preferences [5]. 
Machine learning techniques have shown incredible success in automating normal human intelligence, and can be classified under Algorithm based adaptation as it involves the use of statistical methods to adapt to individual learners with greater specificity [5]. The machine learning algorithms are designed to automatically learn or extract useful patterns from data as a reference for learning styles and learning contents profile from existing data for subsequent classification of learning style or learning content materials. Examples of machine learning algorithms that have been successfully applied in this research domain include Support Vector Machine, Decision Tree, Random forest, among others. In this research, machine learning algorithm is applied to automatically identify learners learning styles based on the learner's browsing history, and to recommend adapted learning material or content to the learner.

\section{RELATED WORKS}

The research work presented by [6] identified the shortcomings of a potential m-learning framework design which uses a proactive approach for retrieving the users' learning contexts without the use of context-aware sensor technologies. The aim of the paper was to construct a theoretical mobile context-aware adaptive learning schedule (mCALS) framework. The methodology follows a proactive approach whereby the learner's schedule retrieves the location and time available contexts. The purpose of the proactive approach was to reduce amount of interactions users are required to input into the devices to inform of their current situation. The research work utilized a 'diary: diaryquestionnaire' research methodology for the usability feasibility which involved the participation of 32 university students. The theoretical framework functionality was examined and analyzed based on three perspectives technical, usability and pedagogical. The paper is focused on providing appropriate learning materials to students based on their current learning contexts. A notable limitation is the constraint of not knowing whether learners are motivated to learn from such a context-aware suggestion mechanism system.

In the research work presented by [2], the research work was motivated by the need to address the adaptation problem by designing a framework which deals with learning content to complement the work in [7] which adapts learning content through the use of mobile technology, based on modelling the learner and all possible contexts related to their current situation. The objective of the paper is to present a framework to describe the factors that play an important role in delivering learning content to mobile learners, and their relationship to each other. The paper proposed a framework for modelling learning content of mobile learning application. The proposed design includes three steps seen as subsystems of the adaptation framework, namely: Creation of the raw Learning content (raw) by the instructor using authoring tools; Development of the learning content structure using meta data labelling which provides details about the learning content and; Learning content classification which involves categorising the learners based on learning styles. Each category has its own learning content representation that facilitates effective learning that matches the style of that learner. This paper presented a new framework that depicts the process of modelling learning content to satisfy individual learner characteristics by taking into consideration his/her context, learning style, application capabilities, learning content. The work was not implemented and thus no evaluation carried out.
"Toward a complete e-learning system framework for semantic" is presented by [8]. This work spurn from the limitations of sole reliance on the course instructor's input on the relationship among the involved concepts which can be imprecise possibly due to the individual biases by human experts. The objective of this paper was to conduct a more thorough e-learning system framework that carefully integrates semantic analysis, concept clustering and learning path optimization. The proposed methodology involves performing an explicit semantic analysis, followed by enhancing the ontology analysis through concept clustering, that is essentially systematic grouping of closely related concepts, and lastly applying an evolutionary optimizer to find an optimal learning path of involved concepts or modules. The evolutionary algorithm was adapted in the work to optimise the learning path with respect to the extracted rule set is fairly standard. To demonstrate the feasibility of the proposed framework, a prototype of the analyser enhanced with concept clustering and rule-based optimizer was implemented. Its performance was compared favourably against the benchmarking shortest-distance optimizer on various actual courses. More importantly, the proposed method clearly demonstrates the importance of enhanced ontology analysis for the overall performance of adaptive or personalized e-learning systems. The proposed framework is limited to achieving optimal learning sequence but not adaptive learning for mobile learners.

An adaptive mobile learning system for the support of personalized learning and device adaptation. The paper identified that it is important and challenging to adapt content so that it satisfies individual demands and fits the requirements of learning devices in a mobile learning environment, hence, learner adaption and device adaption are considered as two important factors to facilitate mobile learning environments for learners with various abilities and learning styles, which this paper seeks to achieve. The objective of this work is to provide learners with an adaptive learning environment according to learner's individual capability and the learning device used. The proposed system exploits Bayesian networks and content adaptation technologies to support both learner adaptation and device adaptation, which allows each learner to construct a personalized and adaptive learning environment. The adaptive mobile learning system architecture is composed of 6 modules namely, user interface module; context detection module; learner profile module; learning diagnosis module; expert knowledge module and content adaptation module. A major contribution to knowledge of this work is that it presents an adaptive mobile learning system (AMLS) that provides learners with adaptive content according to their knowledge levels, learning styles, and heterogeneous learning devices. Also it implored a machine learning technique (Bayesian network) to implement adaptive learning. Further research is required towards improving the inference capability when managing a learning context and arranging content in heterogeneous learning devices.

Modelling and simulation of an adaptive Neuro-fuzzy inference system (ANFIS) for mobile learning is presented by [10]. One of the motivations behind the design of their adaptive mobile learning system was to support the learner throughout the learning activity by realising the adaptation principle, which consists of: capturing the learners' context (implicit and explicit); and then utilizing the captured learner context to construct a Learner Model. This paper presents an Adaptive Neuro-Fuzzy Inference System (ANFIS) for delivering adapted learning content to mobile learners. The 
methodology presents a mobile learning reasoning engine. This reasoning engine provide a suitable learning content format for a mobile learning application, using an Adaptive Neuro-Fuzzy Inference System (ANFIS) based on the acquired learner profile. The proposed architecture was implemented and tested using MATLAB Fuzzy Logic ToolBox. This paper introduced the Adaptive Neuro-Fuzzy Inference System as a reasoning engine to deliver learning content for mobile learning applications. This study was conducted to illustrate the potential effectiveness of ANFIS with hybrid learning, for the adaptation of learning content format for mobile learning users. The performance of ANFIS was evaluated using standard error measurements which revealed the optimal setting necessary for better predictability. The results of the evaluation tests indicate that the most important factors to achieve good performance are usually the training data sample and the number/type of membership functions. The number of epochs and the training options do not appear to have a significant influence on performance. Future research should revise the rules, inputs, number and type of membership functions, the epoch numbers used, and training sample to further refine the ANFIS model. Also there is need to reduce the trade-off between adaptation and time to provide a learner with a personalized profile.

In [11], personalized mobile learning via an adaptive engine is presented. This work is motivated by the need for the system to provide adaptivity in mobile settings based on learners' characteristics and their context. This paper aims to propose an approach for providing personalized course content in mobile settings, considering a combination of students' learning styles and context. The proposed framework is an application that determines the learning style of the user and then provides information in a suitable manner and fashion through mobile technology. Furthermore, context information about the user's environment is considered when providing suitable learning materials. The system architecture consists of several elements, namely: Mobile device-ILS component, Mobile Device-Context Modelling component, Mobile Device-Adaptive Engine, Server, User types and Course material. The course material make up the output component holding output values in either video of lecture, audio recording of lecture, written text of lecture and PowerPoint style of lecture respectively. The proposed approach contributes towards adaptive mobile systems in two ways: First, it aims to demonstrate how information from students' characteristics can be combined with students' current context for providing adaptivity in mobile learning systems. Second, the proposed approach facilitates more detailed investigations on how learners with particular learning styles and in particular contexts use mobile technologies for learning. One identified limitation of this work is implementing the proposed approach, evaluating it within an adaptive mobile system and utilize this system to further investigate students' behaviour and performance in adaptive mobile learning systems. Also need to implore a machine learning approach than using the Index of Learning Style (ILS) questionnaire in capture the learners' behaviour information because the latter would not best capture the learning behaviour of a learner through a question and answer mode.
[12] developed automated adaptive mobile learning system using the semantic web. The authors argued that to the best of their knowledge, there has not been any research that attempts to make the course adaptive in terms of previously completed materials by the students at another educational institution by using the Semantic Web to communicate directly with various educational institutions' systems to acquire the students' profiles. This paper aims to model e-learning and mobile learning systems as a weighted directed graph where each node represents a course unit. In their methodology, an optimal adaptive learning path algorithm is implement utilizing learner information from the learner's profile to enhance specific end goals. The approach demonstrate how to customize the students' profiles by using the Semantic Web in order to provide credit to students for the course units completed in other accredited educational institutions. This work describes the conceptual framework of an Adaptive Mobile Learning System and how the students' profiles are used to adjust the learning path whereby making the learning path more dynamic. This means that when the student learns a course unit, there will be an adjustment to the learning path and a new optimal path will be generated. The architecture of the Adaptive Mobile learning system consists of several modules; System Interface, Student Profile Module (SPM), Learning Style Module (LSM), Domain Concept Module (DCM), Course Content Module (CCM), Learning Path Generation Module (LPM), Student Assessment Module (SAM) and Adaptive Engine Module (AEM). The results from the implementation showed that the Semantic Web can be used with Adaptive Mobile Learning to enhance the courses making them more dynamic. The presented approach is expected to improve the performance of adaptive mobile learning and provides a learning experience to a student that is more personalized and dynamic. This work is limited to only finding optimized dynamic learning path, thus it did not dwell much on learning content adaptation. Need to ensure content adaptation in mobile learning.

A survey on Context Aware and Adaptive Mobile Learning is carried out by [13]. The authors identified that diversity of learners' characteristics as well mobile devices and networks requires personalization for effective adaptive mobile learning. This paper aims to carry out survey about context aware and adaptive m-learning, which serves as the base for new researches in this area present a review of related works on fuzzy based traffic signal control systems. This paper carries out a survey on context aware and mobile learning literatures. The paper subsequently presents the proposed system which uses the clustering technique for generating adapted and personalized content for the user. In this paper, the authors present the overview of mobile learning and importance of context aware and adaptive. The paper also presents the existing works in this domain and point out some salient issues.

\section{PROPOSED METHODOLOGY}

The architecture of the proposed machine learning based framework for adaptive mobile learning using Adaptive Neuro-Fuzzy Inference System (ANFIS) is presented in Figure 1. 


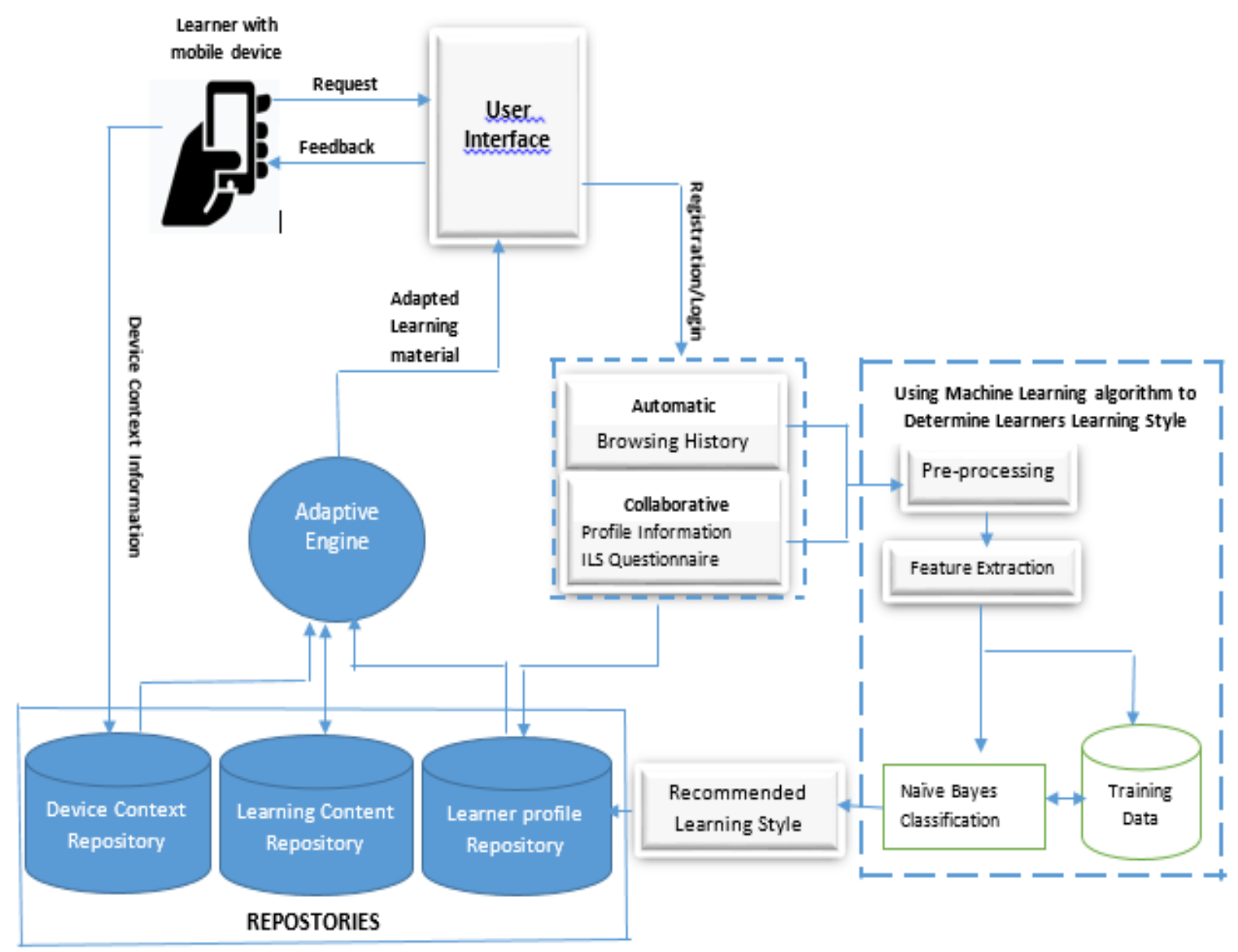

Figure 1: Proposed System Architecture

The conceptual diagram in Figure 1 is composed of two major modules. The first module is client side, while the second module is the server side controlled by a system administrator. Components such as the mobile device, user interface, the learning style module, and the ANFIS engine constitutes the client side, while the server side is composed of device context repository, learning content repository, and the learner profile repository. When a learner with a mobile device make a request for a learning material via the device user interface, the system will mandate the user to register if the user is new to the system. Learners' demography such as name, sex, age, and more, including learning style will be collected and stored in the learner profile repository. The learner's learning style will be determine manually, through the use of questionnaire from Felder-Silverman Learning Style Model (FSLM) or automatically through learner's browsing history (web logs). To automatically determine learner's learning style, learner's $\log$ files will be collected and pre-processed. Thereafter, learner's sequences which are learning objects (videos, audios, pdf, and so on) will be extracted from the preprocessed log files. The extracted learner's sequences will be grouped into FSLM dimensions. Then, Naïve Bayes classifier will be used to predict the leaning style of the learner as proposed in Aissaoui et al., (2019). The adaptive engine used in this research is ANFIS. Here ANFIS will predict Adapted or suitable learning materials by taking into considerations factors such as device context (Network Bandwidth, Battery Life, Screen Size, Software Capabilities, and Processor Speed), environment context (Learner's Location), and learner's context (learning style) as input parameters.

\subsection{Mathematical Model of the proposed model}

Given that the proposed adaptive mobile system $m_{s}$ is composed of a set of objects $L_{s}, D_{T}$, and $A_{m}$ as expressed in equation (1)

$m_{s}=\left\{L_{s}, D_{f}, E_{c,}, A_{m}\right\}$

where, $L_{s} \quad$ denotes $\quad$ Learner's style, $E_{c}$, represents Environmental context such as Learner Location (LL) $D_{f}$ represents Device features such as Network Bandwidth $(N B)$, Battery Life $(B L)$, Screen Size $(S S)$, and Processor Speed (PS), while $A_{m}$ represents Adapted materials (output of the proposed system) expressed in equation (2)

$A_{m}=\{$ text, video, audio, presentation, $p d f\}$

For a learner to access the proposed system, the system creates a profile that contain learner's personal information and learner's style. Felder-Silverman Learner's questionnaire (FSL) is used to manually obtain 8 learner's style which are grouped into four (4) dimensions as follows:

$D=\left\{A / R, S_{n} / I, V_{i} / V_{e}, S_{\text {seq }} / G\right\}$

where $A$ represents Active, $R$ represents Reflective, $S$ denotes Sensing, $I$ represents intuitive $V_{i}$ represents Visual, $V_{e}$ represent Verbal $S_{\text {seq }}$ represent Sequential, and $G$ represents Global. The computation of Felder-Silverman 
expressed in equation (4):

$$
\begin{aligned}
& i(D, q)= \\
D+, \quad & \text { if } \sum q_{\text {end }(A)} \geq \sum q_{\text {end }(B)} \\
D-, & \text { if } \sum q_{\text {end }(A)} \leq \sum q_{\text {end }(B)}
\end{aligned}
$$

where $D+$ represents the values on the left side of each dimensions (i.e, $A, S, V_{i}, S_{\text {seq }}$ ), $D-$ denotes represents the values on the left side of each dimensions (i.e, $R, I, V_{e}, G$ ), $i(D, q)$ indicates the index $i$ of a given pair of dimension, $q_{\text {end }(A)}$ represents the value produced from answering the questions in $D+$, and $q_{\text {end }(B)}$ represents the value produced from answering the questions in $D-$. However, to automatically obtain the learner's style, learner's web history which is stored in web logs will be obtained, preprocessed, and learner's sequences (collection of learning objects accessed by the learning during learning sessions) will be extracted using web usage mining technique. Thereafter sequences of the patterns will be clustered using K-mode algorithm and the clustered sequences will be mapped based on FSLM dimensions. The mapped sequences expressed in equation (5) will serve as training data to Naïve Bayes classification algorithm, which will be used to predict the learning style combination for new sequences.

$Z=\left\{S_{i}, C_{j}\right\} \forall S_{i} \exists f: f=\{1,2,3, \ldots, m\}$

where $Z$ represents the sequence set containing set of sequences $S_{i}, i=1,2,3, \ldots, n$ with a possible learning style $C_{j}, j=\{1,2,3, \ldots, 8\}$, and $f$ set of features (learning objects) that constitute $S_{i}$. Naïve Bayes classifier predicts unknown sequence from the selected feature vector $f=1,2,3, \ldots, p$ using the maximum a posterior. Naïve Bayes classifer is presented as:

$\hat{c}=\operatorname{argmax}_{c_{j}} P\left(c_{j}\right) \prod_{i=1}^{m=16} P\left(f_{i} \mid c_{j}\right), j=1,2,3, \ldots, 8$

where $\hat{c}$ is the predicted learning style and argmax is an argument for Maximum A Posterior (MAP) estimation. After obtaining the learner's style, ANFIS will be used to predict adapted learning material as follows:

ANFIS will require set of input parameters $L L, N B, B L, L_{S}$, and $P S$ with their respective linguistic labels given:

as $L L=\{$ indoor, outdoor $\}, \quad N B=\{$ high, medium, low $\}$, $B L=\{$ high, medium, low $\}, L_{s}=\left\{A, R, V_{s}, V_{b} I, S_{q}, S_{n}\right\}$,

$P S=\{$ high, medium, low $\}$ such that if given the set of input $X_{i}=\left\{L L, N B, B L, L_{S}\right.$, and $\left.P S\right\}$, the node function for each node $i$ is presented as:

$$
\mathrm{y}_{i=1}^{n}=\mu A_{i}\left(X_{i}\right)
$$

where $\mu A_{i}$ is the membership function (Gaussian function) of the linguistic labels $A_{i}$ associated with node $i$, and $\mathrm{y}_{i=1}^{n}$ represents the degree to which input $\mathrm{X}$ satisfies the quantifier $A_{i}$. The Gaussian membership function is computed as follows:

$$
\mu A_{i}\left(X_{i}\right)=\exp \left(-\frac{1}{2} \frac{\left(X-C_{i}\right)^{2}}{\sigma_{i}{ }^{2}}\right)
$$

where $\left(c_{i}, \sigma_{i}\right)$ are parameters to gaussian functions. To compute the firing strength of a rule, every node $i$ multiplies the inputs and send the product out as follows:

$w_{i}=\mu A_{i}\left(X_{1}\right) \times \mu B_{i}\left(X_{2}\right) \times \mu C_{i}\left(X_{3}\right) \times \mu D_{i}\left(X_{4}\right) \times \mu E_{i}\left(X_{5}\right)$

where $w_{i}$ is the firing strength of a rule and $A_{i}, B_{i}, C_{i}, \ldots, D_{i}, E_{i}$ represent linguistic labels for $L L, N B, B L, L_{S}$, and $P S$ respectively. The normalized firing strengths of all the rules is determine by the ratio of the $i$-th rule's firing strength to the sum of all rules' firing strengths as:

$\bar{w}_{i}=\frac{w_{i}}{w_{1}+w_{2}+w_{3}+w_{4}+w_{5}}$

Adaptation is perform with the use of consequent parameters causing every node $i$ to be an adapted node with a node function of the form:

$\mathrm{y}_{i=1}^{n}=\bar{w}_{i} f_{i}=\bar{w}_{i}\left(g_{i} X_{1}+h_{i} X_{2}+k_{i} X_{3}+n_{i} X_{4}+m_{i} X_{5}+p_{i}\right)$

where $f_{i}$ represents ANFIS consequent parameters of learning rate, while $\left\{g_{i}, h_{i}, k_{i}, n_{i}, \ldots, m_{i}, p_{i}\right.$ is the consequent parameter set. The overall output of this model is computed as the weighted average of all incoming input signals and is presented as follows:

$\mathrm{y}_{i=1}^{n}=\sum_{i=1}^{n} \bar{w}_{i} f_{i}=\frac{\sum_{i=1}^{n} w_{i} f_{i}}{\sum_{i=1}^{n} w_{i}}$

The adapted learning material $A_{m}$ will be the output predicted by ANFIS, and will be sent to the Learners' Mobile Device. The model will be implemented using Android Studio.

\section{CONCLUSION}

This research presented a literature reviews on machine learning based adaptive mobile learning system and has established an adaptive Neuro-fuzzy based mobile learning system which takes special considerations of learner's context and device context respectively.

\section{REFERENCES}

[1] Gómeza, S., Zervasb, P. C, Demetrios, G., Sampsonb, C. and Fabregata, R. (2014). Context-aware adaptive and personalized mobile learning delivery supported by UoLmP, Journal of King Saud University - Computer and Information Sciences, Volume 26, Issue 1, Supplement, January, Pages 47-61.

[2] Al-Hmouz, A., and Freeman, A. (2010, June). Learning on location: An adaptive mobile learning content framework. In Technology and Society (ISTAS), 2010 IEEE International Symposium on (pp. 450-456). IEEE.

[3] FernáNdez-LóPez, Á., RodríGuez-FóRtiz, M. J., RodríGuez-Almendros, M. L., \& MartíNez-Segura, M. J. (2013). Mobile learning technology based on iOS devices to support students with special education needs. Computers \& Education, 61, 77-90.

[4] Gómez, S., Zervas, P., Sampson, D. G., \& Fabregat, R. (2014). Context-aware adaptive and personalized mobile learning delivery supported by UoLmP. Journal of King Saud University-Computer and Information Sciences, 26(1), 47-61.

[5] Gavriushenko M., (2017). On Personalized Adaptation of Learning Environment. Ph.D Dissertation from the Faculty of Information Technology, University of Jyvaskyla

[6] Yau, J. Y. K. and Joy, M. (2010) An adaptive contextaware mobile learning framework based on the usability perspective. International Journal of Mobile Learning and Organisation, Volume 4 (Number 4). pp. 378390.doi:10.1504/IJMLO.2010.037535

[7] Alhmouz, A., Shen, J. And Yan, J., (2009). "A Machine Learning based Framework for Adaptive Mobile Learning", The 8th International Conference on Webbased Learning (ICWL 2009), Aachen, Germany, 
published by Springer (LNCS 5686), pp. 34-43.

[8] Tam, V., Lam, E. Y. M., and Fung, S. T. (2012). Toward a complete e-learning system framework for semantic. Proceedings of the 12th ICALT, 592-596.

[9] Huang, H. C., Wang, T. Y., and Hsieh, F. M. (2012). Constructing an adaptive mobile learning system for the support of personalized learning and device adaptation. Procedia-Social and Behavioral Sciences, 64, 332-341.

[10] Al-Hmouz, A., (2012). An adaptive framework to provide personalisation for mobile learners, Doctor of Philosophy thesis, School of Information Systems \& Technology, University of Wollongong, http://ro.uow.edu.au/theses/3465
[11] Tortorella, R. A., and Graf, S. (2012, July). Personalized mobile learning via an adaptive engine. In Advanced Learning Technologies (ICALT), 2012 IEEE 12th International Conference on (pp. 670-671). IEEE.

[12] Hamada, S., Alshalabi, I. A., Elleithy, K., \& Badara, I. A. (2016, April). Automated Adaptive Mobile Learning System using the Semantic WEB. In Systems, Applications and Technology Conference (LISAT), 2016 IEEE Long Island (pp. 1-7). IEEE.

[13] Madhubala, R., and Akila, A. (2017). Context Aware and Adaptive Mobile Learning: A Survey. Advances in Computational Sciences and Technology, 10(5), 13551370. 